HNO $2020 \cdot 68: 473-474$

https://doi.org/10.1007/s00106-020-00860-x

(c) Springer Medizin Verlag GmbH, ein Teil von Springer Nature 2020

J. Dlugaiczyk $\cdot$ D. Labbé

'Deutsches Schwindel- und Gleichgewichtszentrum (DSGZ), Klinikum der Universität München, LudwigMaximilians-Universität, München, Deutschland

${ }^{2}$ HNO-Praxis, 47533 Kleve, Deutschland

\title{
Palliativmedizin in der HNO-Heilkunde
}

\section{Auf die Lebensqualität kommt es an}

\author{
„Man sollte dem anderen die Wahrheit \\ wie einen Mantel hinhalten, damit er \\ hineinschlüpfen kann, und sie ihm nicht \\ wie einen nassen Lappen um die Ohren \\ schlagen." \\ Max Frisch
}

Sehr geehrte Kolleginnen und Kollegen,

die geistige Grundhaltung der Palliativmedizin (pallium, lat.: Mantel) lässt sich wohl kaum treffender beschreiben als mit diesem Zitat von Max Frisch. In diesem Sinne laden wir Sie ein, die Palliativmedizin in der HNO-Heilkunde näher kennenzulernen. Wir danken der Schriftleitung der HNO für die Möglichkeit, ein Leitthemenheft zu diesem wichtigen Bereich unseres Fachgebiets zu entwickeln. Besonderer Dank gilt allen Autoren dafür, dass sie in den hervorragenden Beiträgen ihr umfangreiches Wissen und ihre langjährige Erfahrung mit uns teilen.

Der Eingangsartikel von Herrn Dr. Labbé bietet eine sehr anschauliche und handlungsorientierte Einführung in das Thema unter besonderer Berücksichtigung der im August 2019 erschienenen S3-Leitlinie „Palliativmedizin für Patienten mit einer nicht heilbaren Krebserkrankung“. Ziel des Artikels ist es, die palliativmedizinische Kompetenz eines jeden HNO-Arztes im klinischen Alltag zu stärken - unabhängig von einer speziellen palliativmedizinischen Weiterbildung -, um unseren Tumorpatienten bis zuletzt eine gute Lebensqualität $\mathrm{zu}$ ermöglichen. Neben zahlreichen konkreten Handlungsempfehlungen enthält der Beitrag nützliche Links zu zusätzlichen
Ressourcen und Informationsquellen. Schließlich lädt uns der Artikel ein, eigene Überzeugungen und Glaubenssätze zu überdenken. Lassen Sie sich z.B. von der "surprise question" nach der Indikation für eine palliativmedizinische Mitbetreuung Ihres Patienten überraschen!

Auch der Artikel von Herrn Prof. Dr. Büntzel und Kollegen regt einen palliativmedizinischen Perspektivwechsel an am Beispiel der Ernährungsmedizin bei HNO-Tumorpatienten. Neben praktischen Hilfestellungen für den klinischen Alltag (z.B. Berechnung des täglichen Energiebedarfs eines Patienten) betonen die Autoren insbesondere die Bedeutung von Essen und Trinken über die reine Nahrungsaufnahme hinaus, wie z.B. Genuss, soziale Interaktion, Selbstwirksamkeit. Eine gute palliativmedizinische Ernährungstherapie behält auch diese Faktoren im Blick und liefert so einen wichtigen Beitrag zur Lebensqualität.

\section{》) Oberstes Ziel der \\ Palliativmedizin ist die Verbesserung der Lebensqualität}

Oberstes Ziel der Palliativmedizin ist die Verbesserung der Lebensqualität. Die Gratwanderung zwischen Lebensqualität und Lebenszeit sowie zwischen Ehrlichkeit und Hoffnung steht im Mittelpunkt des Beitrags von Frau Prof. Hübner und Kollegen. Neben den Besonderheiten der Entscheidungsfindung in der Onkologie werden die zentrale Bedeutung der Arzt-Patient-Beziehung und 
der patientenzentrierten Kommunikation in der Palliativmedizin thematisiert. Ehrlichkeit und Empathie sind keine Gegensätze, sondern bilden zusammen den „Mantel der Wahrheit“, in den unser Patient hineinschlüpfen kann - ganz im Sinne des o.g. Zitats von Max Frisch. Es liegt in unserer ureigenen ärztlichen Verantwortung, unserem Patienten diesen Mantel hinzuhalten - in tiefem Respekt vor seiner Individualität und in der Wahrung seiner Autonomie und Würde.

So individuell wie die Patienten sind auch die palliativmedizinschen Therapiekonzepte. Ein wichtiger - wenn auch oft unterschätzter - Baustein innerhalb dieses Konzepts sind chirurgische Maßnahmen. Herr Dr. Sittel und Kollegen erläutern in ihrem Artikel anschaulich Indikationen, Besonderheiten und Grenzen chirurgischer Therapie in der HNO-Palliativmedizin. Oberstes Ziel ist auch hier die Verbesserung der Lebensqualität des Patienten unter bestmöglicher Erhaltung seiner Selbstständigkeit.

Ein weiterer essenzieller Baustein der Palliativmedizin ist die Schmerztherapie, wie Frau Dr. Flöther im abschließenden Beitrag dieses Bandes äußerst verständlich und praxisnah darlegt. Neben übersichtlichen Angaben zum Einsatz und zur Dosierung von Analgetika werden auch die wichtigsten Neben- und Wechselwirkungen sowie Alternativen zur oralen Applikation bei Dysphagie erläutert. Zusammen mit dem Eingangsartikel ermöglicht diese Übersicht jedem HNOArzt, eine Basis-Schmerztherapie bei seinen Tumorpatienten durchzuführen.

Wir hoffen, dass dieses Leitthemenheft Sie dabei unterstützt, Ihren Tumorpatienten eine frühzeitige palliative Mitversorgung zu ermöglichen, und Sie da$\mathrm{zu}$ ermutigt, palliativmedizinische Elemente in Ihre eigene ärztliche Tätigkeit aufzunehmen. Wir freuen uns über Ihre Rückmeldungen, Anregungen und Erfahrungsberichte zu diesem Thema.

Mit bestem Dank für Ihr Interesse und freundlichen kollegialen Grüßen

Julia Dlugaiczyk und Daniel Labbé

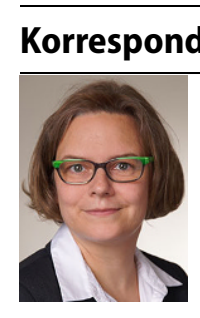

Prof. Dr. med. J. Dlugaiczyk Deutsches Schwindel- und Gleichgewichtszentrum (DSGZ), Klinikum der Universität München, Ludwig-MaximiliansUniversität

Marchioninistraße 15, 81377 München, Deutschland julia.dlugaiczyk@gmx.de

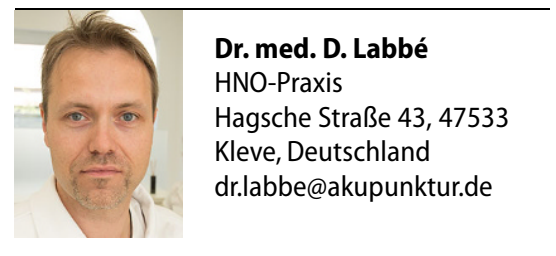

Interessenkonflikt. J.Dlugaiczykund D. Labbégeben an, dass kein Interessenkonflikt besteht.

\section{Leitthemenübersicht HNO 2020}

\section{HNO}

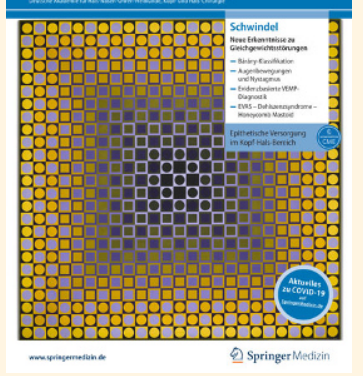

HNO bietet Ihnen umfassende und aktuelle Beiträge zu interessanten Themenschwerpunkten aus allen Bereichen der Hals-, Nasen- und Ohrenheilkunde, Kopf- und Halschirurgie sowie Allergologie und ihren verwandten Gebieten.

\section{0}

- 01/20 Originalien

- 02/20 Immunonkologie

- 03/20 Geriatrie

- 04/20 Ausbildung, Lehre, Weiterbildung

- 05/20 Schwindel

- 06/20 Pädiatrische HNO-Heilkunde

- 07/20 Palliativmedizin

- 08/20 Kieferhöhle

- 09/20 Onkologie Teil 1

- 10/20 Preisträger

- 11/20 Lehre, Weiterbildung in der HNO

- 12/20 Onkologie Teil 2

(Änderungen vorbehalten)

Einzelne Ausgaben können Sie direkt bei unserem Kundenservice zum Preis von je EUR 44,- zzgl. Versandkosten beziehen. So erreichen Sie unseren Kundenservice:

Springer Customer Service Center GmbH Kundenservice Zeitschriften Tiergartenstr. 15, 69121 Heidelberg

Tel.: +49 $6221345-4303$

Fax: +49 $6221345-4229$

leserservice@springer.com

www.springermedizin.de/hno-zeitschrift 